many of the completed forms as you can personally and draw the interviewers' attention to the problems. If you cannot identify any, look again: you have simply missed them. At least one of the questions will turn out to be hopeless despite steps 1 to 8 ; this is par for the course and not worth losing sleep over. Take pains to achieve a high response rate, especially in postal surveys. If the response rate is poor and you are confident that your questionnaire is not to blame send out a reminder along with a second and even a third copy of the form. Avoid haranguing non-respondents but emphasise to them how important their cooperation is to the success of your extraordinarily important study. Flattery works.

(10) Start again - Good research is usually the result of learning from mistakes. If time and resources (as well as personal motivation) permit replicate your study at least once. This will allow you to perform validation, to increase the sample size, and to fine tune your questionnaire to the point where you can be proud of it.
You now know about as much theory as you need to get you started on your survey. For those with the time and inclination, more detailed advice on questionnaire design can be found in good medical libraries. ${ }^{67}$ Some aspects of the subject may appear highly technical and complicated to the point where you may be deterred from going further. As there is no substitute for experience, however, take a deep breath and jump. The landing will not be as hard as you fear.

1 Bennett $\mathrm{AE}$, Ritchie $\mathrm{K}$. Questionnaires in medicine. A guide to their design and use. London: Oxford University Press (for the Nuffield Provincial Hospitals London: Oxford

2 Hulley SB, Cummings SR, eds. Designing clinical research. Baltimore: Williams and Wilkins, 1988 .

3 McDowell I, Newell C. Measuring health. A guide to rating scales and questionnaires. New York: Oxford University Press, 1987.

4 Eastwood RP. Sales control by quantitative methods. New York: Columbia University Press, 1940.

5 Cartwright A. Health surveys in practice and in potential: a critical review of their scope and methods. London: King Edward's Hospital Fund, 1983.

6 Sudman S, Bradburn NM. Asking questions. London: Jossey Bass, 1982

7 Streiner DL, Norman GR. Health measurement scales. A practical guide to their development and use. Oxford: Oxford University Press, 1989.

(Accepted 23 August 1993)

\title{
Minimally Invasive Surgery
}

\section{General surgery: biliary surgery}

\section{R C G Russell}

This is the first in a series of articles on minimally invasive surgery

\section{Middlesex Hospital, London W1N 8AA R C G Russell, consultant surgeon}

BMF 1993;307:1266-9
The management of biliary tract disease has changed completely as a result of minimally invasive treatment. For most patients with gallstones that cause symptoms a laparoscopic cholecystectomy will treat the condition with minimal morbidity and a short recovery period. If complications are encountered, conversion to a mini-cholecystectomy gives results that are nearly as good. Acute cholecystitis can be treated by percutaneous drainage followed either by percutaneous cholecystolithotomy or a laparoscopic cholecystectomy. Gallstones in the bile duct are best treated by endoscopic sphincterotomy with duct clearance. The day of the large cholecystectomy scar with its subsequent incisional hernia has gone.

The concepts of minimally invasive surgery in the biliary tree grew out of the role of the endoscopist in treating gallstones in the bile duct by endoscopic sphincterotomy. From then on the search for a minimally invasive technique to deal with stones in the gallbladder took many turns. Techniques to intubate the gallbladder endoscopically proved difficult, and it is still only rarely possible to negotiate the valves of the cystic duct to gain entry to the gallbladder. A better approach proved to be a percutaneous transhepatic puncture of the liver with the insertion of a catheter, through which solvents could be injected and stones dissolved. This proved time consuming and technically difficult, and the operation has been largely abandoned. For draining an acute empyema of the gallbladder, however, percutaneous drainage can be a dramatic lifesaving and non-invasive technique.

It became apparent that to gain access to the gallbladder and remove the stones it was necessary to pass larger catheters into the gallbladder, and thus the technique of percutaneous nephrolithotomy was adapted to the gallbladder. This technique proved successful in removing the gallstones, but a drain had to be left in the gallbladder to allow the gallbladder to heal round it so that bile did not leak into the peritoneal cavity on its removal. This was done 10 days later, after the biliary tree had been checked radiologically to ensure that there were no residual stones. The major disadvantage of this technique is that up to a third of patients are subject to formation of new stones in the gallbladder, and more than half of these patients have to have their gallbladder removed within three years of the original procedure.

After trials with oral dissolution, contact dissolution, extracorporeal lithotripsy, percutaneous cholecystolithotomy, and rotary lithotripsy attention is now focused almost entirely on cholecystectomy for the management of gallstones either by the laparoscopic technique or by mini-cholecystectomy. Endoscopic retrograde cannulation of the bile duct remains pre-eminent as the method of dealing with a gallstone in the bile duct by minimally invasive technology.

\section{Laparoscopic cholecystectomy}

The standard treatment for gallstones in developed countries is laparoscopic cholecystectomy. This treatment was first described in Germany in 1985 but was published in an obscure journal and received little public acclaim. Mouret in Lyons, who is both a general and a gynaecological surgeon, performed the first publicised laparoscopic cholecystectomy in March 1987. Dubois in Paris, who for a long time had been adept at minicholecystectomy, progressively replaced this approach with laparoscopic cholecystectomy from February 1988. ${ }^{1}$ In June 1988 McKernan and Saye performed the first laparoscopic cholecystectomy with a laser to dissect the gallbladder. ${ }^{2}$ This technique developed rapidly under the stimulus provided by Reddick in Nashville, Tennessee, from October 1988.

The world at large became familiar with the technique when Perrisat from Bordeaux presented a video of it to the Society of American Gastrointestinal Endoscopic Surgeons in April 1989. ${ }^{3}$ By the spring of 1990 the operation was performed in numerous centres in the United Kingdom. In 1992 over $60 \%$ of cholecystectomies performed in the United Kingdom were done by the laparoscopic method. 
INDICATIONS FOR SURGERY

In the past year the number of cholecystectomies performed in the United Kingdom and worldwide has increased from the $600-800$ procedures per million population per year that had been undertaken, with slight variation from region to region in previous years. It is tempting to suggest that the recent rise is due to an entrepreneurial effort to learn and practise a new technique that is of interest to the technically oriented surgeon. No surgeon would deny such temptation, but the public is not blameless: patients who previously put up with an occasional attack of biliary colic suddenly appear to be keen to have the minor procedure, and patients who have just had their first attack are keen to have the new operation in case severe inflammation makes the old operation necessary. I anticipate that the present rate of operations will settle down to the previous level or to an even lower number if present dietary trends continue.

The prime indication for cholecystectomy is biliary colic, with its characteristic and well defined pain. Acute cholecystitis, with its complications, is an urgent indication for cholecystectomy. Indeed, an attack of gallstone related disease necessitating admission to hospital is an indication for cholecystectomy during that admission or soon after to prevent more severe symptoms or complications developing. Jaundice due to gallstones is a further indication for cholecystectomy after the gallstones have been treated endoscopically except in elderly patients, for whom the endoscopic procedure is sufficient in $85 \%$ of cases. Patients with non-specific dyspepsia or symptoms suggestive of the irritable bowel syndrome should only be offered cholecystectomy with caution. Rigid avoidance of the procedure with such patients could deny some sufferers effective treatment, but patients should be clearly told that some symptoms may remain after the operation. There is no such thing as the postcholecystectomy syndrome, but the incidence of gastrointestinal symptoms after the operation will be very high if unsuitable patients are given cholecystectomy. It can now be stated dogmatically that symptomless gallstones do not require treatment.

There are situations when a surgeon may be unwilling to undertake laparoscopic cholecystectomy. When the technique was first developed it was thought to be inadvisable in patients who were pregnant, grossly obese, or who had just recovered from acute pancreatitis. Ideas have changed, and these conditions are now considered ideal for the laparoscopic technique. Many surgeons with a keen interest in laparoscopic surgery will first consider the laparoscopic approach for all patients who require a cholecystectomy. Attempts have been made to identify patients who are unsuitable for the procedure by preoperative ultrasonography: it is known that patients with a thick walled gallbladder, a large stone or evidence of inflammation in Hartmann's pouch, or a localised abscess will prove difficult, but the laparoscopic approach can still be undertaken in most of these patients.

\section{SURGICAL TECHNIQUE}

Preoperative preparation-Patients should undergo preoperative ultrasonography for examination of the gallbladder and bile duct. If the gallbladder is highly inflamed or has thickened walls or there is pus present the patient should be warned that conversion to open cholecystectomy may be necessary. Similarly, if the common bile duct is more than $5.5 \mathrm{~mm}$ in diameter a preoperative endoscopic cannulation of the bile duct should be performed to remove any stone present. Patients with tests showing abnormal liver function and with a history of jaundice should probably have preoperative endoscopy. Many centres also undertake intravenous cholangiography before laparoscopic cholecystectomy to assess anatomy. Patients must be assessed by an anaesthetist to ensure that they are fit for general anaesthesia and a pneumoperitoneum with a pressure of $15 \mathrm{~mm} \mathrm{Hg}$. Anaesthetised patients are placed on the operating table either flat or in a modified lithotomy position so that the surgeon can stand between the legs.

Equipment-For a pneumoperitoneum a high flow carbon dioxide insufflator, preferably with a facility for recirculating the carbon dioxide through a filter to remove smoke, is needed. Adequate safety alarms must be attached to the insufflator so that excessive pressures of carbon dioxide cannot occur in the abdomen. As diathermy is used for much of the dissection and is the main method of stopping haemorrhage, an efficient coagulation diathermy machine is important. Means of suction and of flushing the peritoneal cavity with saline are necessary to ensure a field free of blood and other fluids such as bile. A video camera with its attached light source is vital because accurate surgery cannot be undertaken without clear vision. The new three chip cameras attached to high definition television monitors greatly ease the dissection and overcome the problems associated with two dimensional as opposed to three dimensional vision. A backup facility for each piece of apparatus is needed in case of failure.

Gaining access-Two Kochers forceps are placed on the skin of the umbilicus, which is everted, and a transverse incision is made in the umbilicus. A Verres needle attached to the carbon dioxide insufflator is passed into the incision, the Kochers forceps are lifted up, and the needle is passed through the linea alba and peritoneum into the peritoneal cavity. The formation of the pneumoperitoneum can then be started, and insufflation continues until the pressure in the intraabdominal cavity reaches $15-17 \mathrm{~mm} \mathrm{Hg}$. A trocar and a cannula are then placed through the same umbilical incision into the abdominal cavity. I prefer nondisposable trocars as the cost of the disposable trocar makes the procedure uneconomic. The laparoscope, to which the video camera is attached, is advanced into the peritoneal cavity, and the gallbladder and peritoneal cavity are examined. Provided that the gallbladder seems suitable for laparoscopic removal, a $10 \mathrm{~mm}$ epigastric trocar is inserted through the linea alba to enter the peritoneal cavity just to the right side of the falciform ligament, and $5 \mathrm{~mm}$ trocars are placed laterally near the subcostal margin and in the midclavicular line, preferably at the level of the umbilicus, to provide good triangulation between the three working ports.

Freeing the gallbladder-Grasping forceps are put into the lateral trocar and placed on the fundus of the gallbladder, which is then lifted up with the liver to expose the rest of the gallbladder and the subhepatic space (fig 1). Adhesions are dissected away from the gallbladder to expose the whole organ. The second grasping forceps is placed on Hartmann's pouch and pulled downwards to expose the triangle of Calot. The diathermy hook is passed through the epigastric cannula, and a careful inspection of the anatomy is made to define the position of the duodenum, the common bile duct, and the artery and its cystic duct branch. The peritoneum at the lower border of the gallbladder is then divided both anteriorly and posteriorly from the cystic duct to the liver bed (fig 2). These dissections are deepened until the branches of the cystic artery are found (fig 3): the cystic artery almost always breaks up into numerous branches before reaching the gallbladder. Individual branches can be coagulated by diathermy, or the main trunk can be clipped. The principle of this dissection is to remain as close to the gallbladder as possible. When the triangle of Calot has been completely exposed and the 


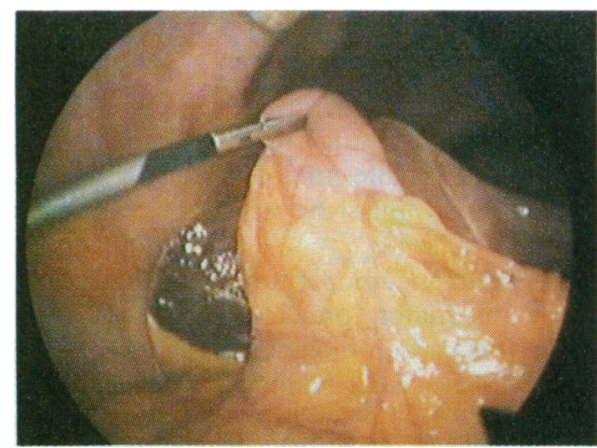

FIG 1-Fundus of gallbladder is lifted up to show omentum attached to lower border of slightly inflamed gallbladder

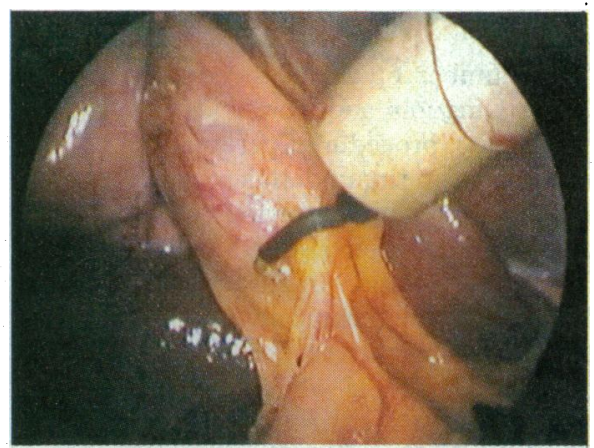

FIG 2-Adhesions separated by hook diathermy and now duodenum being separated from gallbladder by division of adhesions

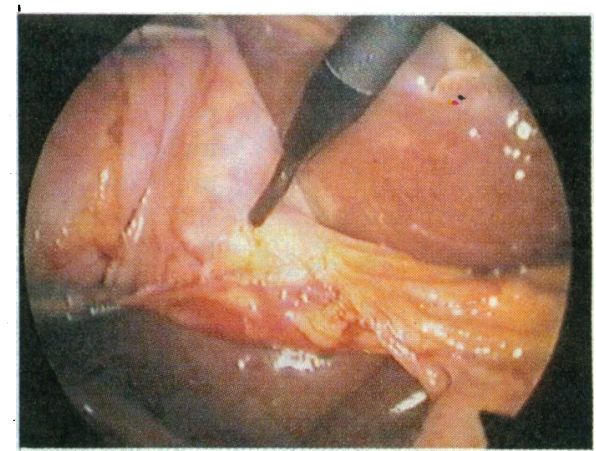

FIG 3-Triangle of Calot being exposed by dividing peritoneum over lower border of gallbladder away from bile duct and on gallbladder wall diathermy hook can be passed from the front dissection to the back dissection the cystic duct is cleared of tissue so that it can be clipped (fig 4). At this stage peroperative cholangiography can be done. Only $50 \%$ of surgeons think that this is necessary because by this stage of the operation the main anatomy has been defined and if the triangle of Calot has been completely exposed there is little danger of damage to the common hepatic or right hepatic ducts. When the cystic duct has been exposed two absolok clips are placed near to the common bile duct and a ligaclip is placed near to the gallbladder (fig 5). The cystic duct is divided, and the gallbladder is then dissected from the gallbladder bed until it is free of all attachment to the liver. Some surgeons do this by laser, but diathermy is adequate and much cheaper.

Extracting the gallbladder-The gallbladder is extracted through the umbilicus (fig 6). If the gallstones are large these have to be removed separately through the umbilicus with De Jardin forceps. Large stones and big or thick walled gallbladders can provide technical difficulties, especially if the gallbladder has been torn or punctured during a tedious dissection. The preferred technique is to place the gallbladder in a specially constructed bag, which is brought out through the umbilical incision, and to break up the stones with forceps in the bag so that the debris can be removed without contamination of the peritoneal cavity. When the gallbladder has been extracted gas is sucked from the abdomen, and the wounds are closed with a 5/0 subcuticular suture.

Conversion to open surgery-Probably the most difficult technical situation which the laparoscopist encounters is bleeding. If the cystic artery or the right hepatic artery is damaged the application of clips in the absence of clear vision can lead to the inappropriate application of the clip to the common bile duct Similarly, diathermy without clear anatomical definition can cause damage to the biliary tree and subsequent formation of a fistula. A large stone or gross inflammation within the triangle of Calot can lead to a difficult dissection so that it is almost impossible to dissect the cystic duct safely. If any difficulty is encountered during the procedure conversion to a mini-cholecystectomy is safe, proper, and appropriate: persisting for many hours trying to dissect difficult anatomy is not. The differences between mini-cholecystectomy and laparoscopic cholecystectomy are so small that excessive operative times can no longer be justified. A conversion rate of $5 \%$ is acceptable, and higher and lower rates are probably equally inappropriate.

Postoperative care-Recovery is rapid after this procedure, and the patient is usually up on the evening of the day of the operation and drinking normally. Intravenous fluids are stopped either on the evening after the operation or the following morning. More than half of patients leave hospital within 24 hours of the operation while the rest leave within three days. Complications are rare if the procedure is done carefully, but if a complication is suspected immediate ultrasonography of the abdomen should be undertaken to look for collections of fluid in the subhepatic space or bleeding. If fluid is seen a drain should be inserted percutaneously with ultrasound or $x$ ray guidance. If bile is found in the drainage fluid an endoscopic cannulation of the bile duct should be done within 24 hours. If contrast medium leaks from the stump of the cystic duct during endoscopic cholangiography it may be due to the clip slipping or to a small biliary radical in the gallbladder bed, but endoscopic sphincterotomy invariably solves the problem. Narrowing of the bile duct requires immediate balloon dilatation or stenting to salvage a difficult situation, while if there is transection of the duct the appropriate operative procedure is indicated. Bleeding is rare after this operation, and in my experience it can usually be managed conservatively. Wound infections are minor and rarely cause problems.

\section{OUTCOME}

Critics of this operation point to the large number of complications that, according to hearsay, occurs with this procedure. There is now, however, good evidence

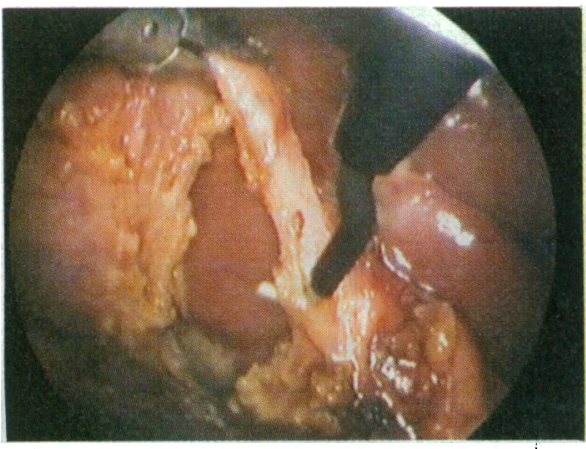

FIG 4-Triangle of Calot widely opened and cystic duct being cleared of tissue

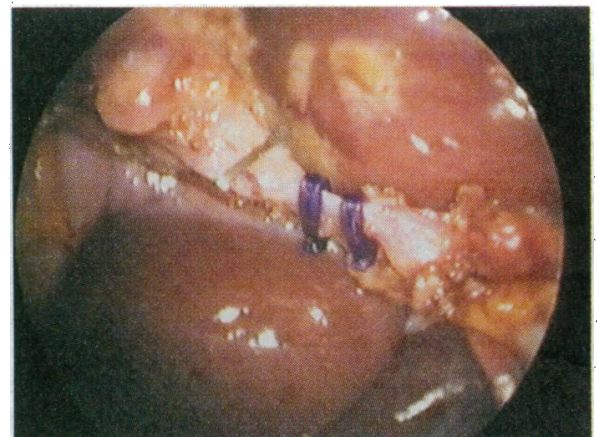

Fig 5-Cystic duct now cleaned and twoo absolok clips placed proximally with a ligaclip on gallbladder

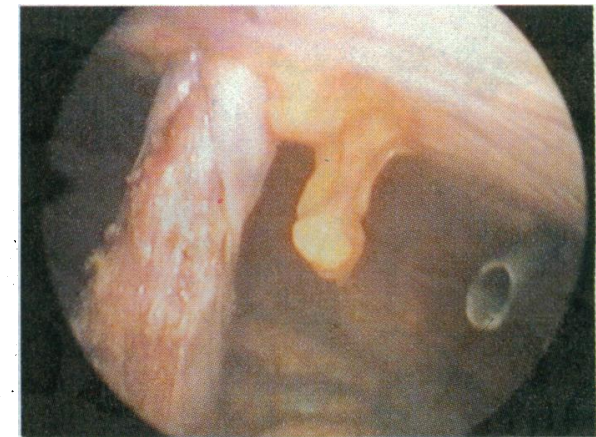

FIG 6-Freed gallbladder being pulled out through umbilicus 
from Europe and the United Kingdom that the rate of complication of the laparoscopic method is at least as good as that of open cholecystectomy if not better. A recently published American review of 77600 cases reported that laparotomy for the treatment of a complication was required by $1.2 \%$ of patients. ${ }^{4}$ The mean rate of bile duct injury was $0.6 \%$, and these injuries occurred mainly during the first 100 cases. Postoperative bile leak was recognised in $0.3 \%$, and only 18 of the 33 postoperative deaths resulted from operative injury. Furthermore, the long term rate of complication was reduced by the low number of complications associated with the wound, such as infection and incisional hernia. As already mentioned, patients return to work much more rapidly than after open surgery.

\section{Gallstones in the bile duct}

The management of stones in the common bile duct is still a controversial subject. About $5 \%-10 \%$ of patients who have a cholecystectomy will have a stone in the bile duct. This may present with jaundice or be diagnosed at the time of cholecystectomy by operative cholangiography. Only a half of surgeons regularly perform operative cholangiography at cholecystectomy; a quarter undertake cholangiography selectively, usually because of a history of jaundice, the presence of an enlarged bile duct, or a test showing abnormal liver function; and a quarter never perform the procedure. If a gallstone is found by cholangiography it can either be left for the patient to undergo an endoscopic sphincterotomy later, or the stone can be removed during the laparoscopic procedure. One method is to dilate the cystic duct and then either see and retrieve the stone by means of a cholangioscope passed through the hole in the cystic duct or remove the stone by basketing techniques with radiological guidance. Alternatively, some surgeons directly incise the duct as at open surgery and remove the stone. This technique has the advantage that the whole duct can be explored more easily, but there is more contamination and the defect has to be sutured, which is difficult to perform laparoscopically.

Others argue that an endoscopic sphincterotomy is preferable. This technique is safe in competent hands, it is thorough in that good clearance of the whole duct is achieved, and it prevents stasis within the bile duct leading to formation of another stone. Initial worries about the long term effects of sphincterotomy have so far proved groundless.

\section{Extracorporeal shock wave lithotripsy}

Extracorporeal shock wave lithotripsy was pioneered in Munich and was first used in 1980 to treat kidney stones. ${ }^{5}$ Experimental work suggested that this technique would also fragment gallstones. ${ }^{67}$ In the procedure shock waves are generated, focused, and transmitted to the body through a liquid medium, usually water, that has an acoustic impedance similar to that of body tissues. The shock waves spread through the body with little energy loss and therefore minimal tissue damage. The stone must be accurately positioned at the focal point of the waves for the release of the high energy on impact, which creates mechanical stresses and leads to fragmentation of the stone. After fragmentation of the gallstone in the gallbladder the spontaneous discharge of stone fragments is unlikely to be complete. These fragments must negotiate the cystic duct and its valves of Heister, the common bile duct (which has no peristalsis), and the sphincter of Oddi to reach the duodenum. Emptying of the normal gallbladder is slow and incomplete, and in patients with gallstones it is considerably impaired. Adjuvant treatment is therefore given in the form of oral bile acids to dissolve the fragments and to allow them to negotiate the pathway to the intestine. The best results suggest that $75 \%-95 \%$ of stones are fragmented completely, but it takes up to 18 months for $91 \%$ of patients to become clear of stones. ${ }^{8}$ The limited information on recurrence of gallstones suggests that a third of patients will have a recurrence in three years. This technique is probably not useful for managing gallstones, and the rare instance of a stone stuck in the common bile duct, which is difficult to remove by endoscopic sphincterotomy, is likely to be its only indication.

\section{The acute gallbladder}

Laparoscopic cholecystectomy is difficult in the presence of complications such as empyema or perforation, and in elderly and unfit patients with acute cholecystitis a laparoscopic or open approach may be dangerous. Minimally invasive treatment can solve the problem of acute cholecystitis: a puncture of the acute gallbladder can easily be made with ultrasound guidance and with minimal upset to a patient under local anaesthesia. Once a needle is in place a guide wire can be inserted, and a drain can be placed over the guidewire. After an acute gallbladder has been drained there is usually immediate resolution of symptoms, and the systemic effects resolve rapidly. It is then possible to proceed to laparoscopic cholecystectomy or, if the patient is unfit or elderly, percutaneous cholecystolithotomy under local anaesthesia. With $x$ ray guidance the tract is dilated with a 9 or 12 French gauge catheter, a cholangioscope is pushed into the gallbladder, and the stones are shattered by means of contact lithotripsy. The gallbladder is then flushed out to completely remove the stone fragments. A small drain is inserted for repeat cholangiography two to three days later, and if the gallbladder and biliary tree are clear of stones the drain is removed. Most patients will have no further trouble from their gallbladder, which will shrink to a small size.

1 Dubois F, Icard P, Berthelot G, Levard H. Coelioscopic cholecystectomy. Preliminary report of 36 cases. Ann Surg 1990;211:60-2.

2 McKernan JB, Saye WB. Laparoscopic general surgery. 7 Med Assoc Ga 1990;79:157-9.

3 Perissat J, Collet DR, Belliard R. Gallstones: laparoscopic treatment, intracorporeal lithotripsy followed by cholecystostomy or cholecystectomy-a personal technique. Endoscopy 1989 [suppl 1];21:373-4.

4 Deziel DJ, Millikan KW, Economou SG, Doolas A, Ko S-T, Airan MC. Complications of laparoscopic cholecystectomy: a national survey of 4,292 hospitals and an analysis of 77,604 cases. Am 7 Surg 1993;165:9-14.

5 Chaussy C, Schmiedt E, Jocham D, Brendel W, Forssmann B, Walther V. First clinical experience with extracorporeally induced destruction of kidney clinical experience with extracorporeally in

6 Brendel W, Enders G. Shock waves for gall stones: animal studies. Lancet 1983;i:1054.

7 Sauerbruch T, Delius M, Paumgatener G, Holl J, Wess O, Weber W, et al. Fragmentation of gallstones by extracorporeal shock waves. $N$ Engl $f$ Med 1986;314:818-22.

8 Sackman M, Delius M, Sauerbruch T, Holl J, Weber W, Ippisch G, et al. Shock wave lithotripsy of gallbladder stones. The first 175 patients. $N$ Engl $\mathcal{f}$ Med 1988;318:393-7.

\section{Correction}

Arthroscopic surgery compared with supervised exercises in patients with rotator cuff disease (stage II impingement syndrome)

A typesetting error and an editorial error occurred in this paper by Jens Ivar Brox and colleagues (9 October, $p$ 899). In table III the median scores for function at 6 months should have read 21.0 for placebo laser treatment and 26.0 for supervised exercises (not $15 \cdot 0$ and 25.0 , respectively, as published). The third sentence of the third paragraph of the discussion should have read: "Verbal pain ratings as part of the Neer shoulder score were always made during the clinical examination, but patients scored the pain they had experienced during the previous week on nine point scales before the examination. Verbal pain ratings may therefore have been biased." 